obvious spin-off. The integration of other detailed or local geological data-sets of different type (such as metallogenic) is another new possibility beckoning the Gondwana researcher.

The Reunite Gondwana project therefore seems set to lead from map to database and colour-graphics workstation display, from static picture to kinematic and selectively multi-faceted imagery. But although the wall-map may not have the

\section{Reading palms}

\section{Peter H. Raven}

Genera Palmarum: A Classification of Palms Based on the Work of Harold $E$. Moore, Jr. By Natalie W. Uhl and John Dransfield. The L.H. Bailey Hortorium/ International Palm Society: 1987. Pp. 610. Distributed by Allen Press, Lawrence, Kansas. \$74.95. (Available in Britain from the Bentham-Moxon Trust, Royal Botanic Gardens, Kew, $£ 45$ plus postage.)

PALms are second only to the grasses in worldwide economic importance and they dominate ecosystems throughout the warmer parts of the globe. A lifetime of work by Harold Moore, who died prematurely in 1980 , provided the basis for the new classification presented in this volume. Although primarily taxonomic in scope, the book is so attractively presented and well organized that it assumes a general importance one would not necessarily associate with such a work.

In writing the book, the authors did not simply take over Moore's concepts and expand on the material available in his notes. Instead, they methodically reviewed all genera of palms themselves, reassessing Moore's views and often bringing their conclusions into better agreement with the evidence. In doing so, they drew upon their own considerable studies of the group, and were often able to improve greatly on the concepts available in 1980 . Pointing out several areas where our knowledge remains inadequate for a definitive classification, the authors call for cladistic studies of the palms to improve both the classification and our understanding of evolutionary patterns in the family. It is also clear that the application of macromolecular studies to the further elucidation of relationships in the family could be undertaken with profit, using the classification presented here as a starting point.

Although some of the habit photographs could have been better, the way in which this volume presents the diversity of palms - plants that can be appreciated properly only in a living state - is truly impressive. In this connection, special mention must be made of Marion Ruff gee-whiz appeal of an electronic product, it will undoubtedly trigger intense study and reflection on some of the salient problems of how the Earth works. It deserves a prominent place in every Earth scientist's office, and in the foyers of all geological institutions.

C.J.H. Hartnady is in the Precambrian Research Unit, Department of Geology, University of Cape Town, Private Bag, Rondebosch 7700 , South Africa.

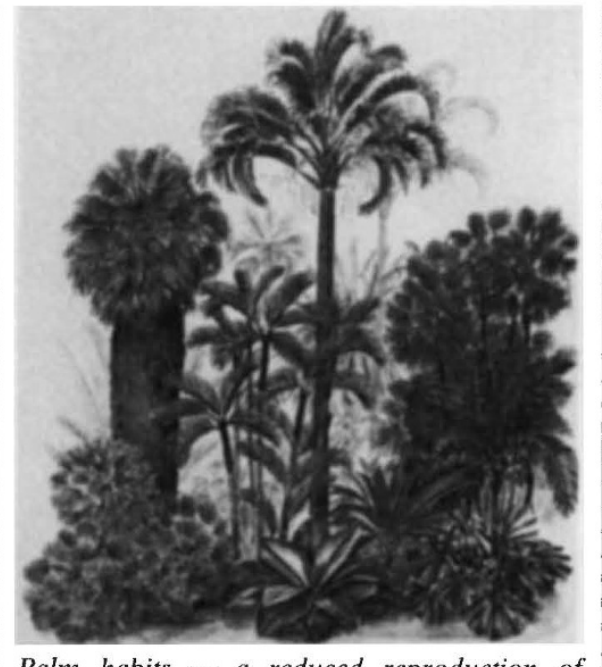

Palm habits - a reduced reproduction of Marion Ruff Sheehan's colour original.

Sheehan's superb illustrations, which demonstrate the value of having an artist work consistently on a project of this sort.

In this classification, palms are divided into 200 genera. Thorough morphological and anatomical investigations of vegetative features, inflorescences, pollen, chromosome numbers, ecology and geography, based on travel throughout the tropics and subtropics, have led to the grouping of these genera into six subfamilies and 16 tribes. The descriptions of the genera are clear and well presented, and there are ample diagnostic illustrations. A particularly useful innovation is the well-illustrated glossary, which imparts unusual clarity to the meaning of the descriptions.

In terms of species, palms constitute only about 1 per cent of the estimated 250,000 flowering plants. In terms of their role in the ecosystems of all warmer regions, however, they are much more important than that number would seem to signify. Because of that vital ecological role, it is especially gratifying that palms are the subject of such a landmark work. Genera Palmarum will stand as a key work of reference for decades to come, and it establishes a new standard for monographic treatments of the major plant groups. One hopes that it will be emulated often in years to come.

Peter H. Raven is Director of the Missouri Botanical Garden, PO Box 299, St Louis, Missouri 63166, USA.

\section{With a little bit of magic}

\author{
M.J.A. Tanner
}

Immunochemical Methods in Cell and Molecular Biology. By R. J. Mayer and J. H. Walker. Academic: 1987. Pp.325. $£ 26, \$ 49.50$.

Books describing practical techniques in the biochemical sciences are becoming increasingly popular, both because of the diverse range of methods in use nowadays and because pressure on space in journals rarely allows the inclusion of more than a bare outline of experimental protocols in papers.

Mayer and Walker's volume covers the range of methods which use antibodies that are now established as basic tools in biological research. The major portion of it consists of a review of the production and applications of both polyclonal and monoclonal antibodies, with sections on most of the immunochemical techniques in common use. The authors assume little previous knowledge of the subject and provide a very readable discussion of the topic.

There is still a little bit of magic in preparing good antibodies and getting them to work well. The empirical basis of many immunochemical methods often makes it difficult to define clearcut rules in applying them, and this is reflected in the book. The survey discusses the methods mainly by example, many of which are taken from the authors' own work. It also provides a useful summary of the variety of experimental conditions which have been employed with each of the techniques discussed. The review concludes with a chapter on synthetic peptides as antigens, which is written with great enthusiasm but unfortunately does not cover some of the newer developments in adjuvants for raising anti-peptide antibodies.

The final part of the book contains a collection of step-by-step experimental details for most of the methods described in the review. Surprisingly, no protocols for the preparation of monoclonal antibodies are provided, although the review does contain some relevant experimental guidance. Also omitted are details of the faster and more economical dry and semidry electroblotting techniques. But this part of the book provides a clear and detailed guide to a large number of methods, ranging from the raising of antibodies to immunohistochemistry on tissue sections. It will be a useful laboratory manual of immunochemical techniques, especially for those unfamiliar with the area.

M.J.A. Tanner is a Reader in the Department of Biochemistry, School of Medical Sciences, University of Bristol, Bristol BS8 ITD, UK. 